\title{
Adaptações fisiológicas de arraias Potamotrygon Rex (Potamotrygonidae) frente às variações sazonais no ambiente
}

As espécies de arraias de água doce (família Potamotrygonidae) têm sua história evolutiva intrinsecamente conectada à história geológica das bacias hidrográficas neotropicais. Os representantes desse grupo acumulam uma série de adaptações fisiológicas que possibilitam a manutenção de suas populações em condições ambientais variáveis, sendo a maioria dessas ainda desconhecidas. Assim, este trabalho teve por objetivo analisar as respostas fisiológicas das arraias Potamotrygon rex, frente às variações do ambiente, ocorridas durante o ciclo hidrológico (períodos chuvoso/estiagem) na região do Alto/Médio rio Tocantins. $\mathrm{O}$ ambiente fo verificado na tomada de amostras da água durante sete semanas consecutivas, no período chuvoso e de estiagem, na mesma região onde foram coletadas as arraias. Os espécimes foram coletados na área de transição do reservatório do Lajeado, no rio Tocantins, dos quais foram registrados os dados biométricos e coletadas amostras de sangue para verificação dos indicadores fisiológicos (hematócrito, hemoglobina e glicose plasmática). Foi calculado o fator de condição relativo das arraias (Kn), e a relação ente os dados biométricos e as variáveis fisiológicas foram avaliada pela correlação de Pearson. Como resultado, verificou-se as alterações ambientais que ocorrem sazonalmente, durante o ciclo hidrológico (períodos chuvosos e estiagem). No período chuvoso (de outubro a abril), a temperatura da água $\left({ }^{\circ} \mathrm{C}\right)$, o oxigênio dissolvido na água $(\mathrm{mg} / \mathrm{L})$ e a turbidez apresentaram valores mais elevados do que no período de estiagem (de maio a setembro), com exceção da condutividade elétrica. Para os espécimes coletados na estiagem verificou-se o aumento do peso, do Kn, do hematócrito, hemoglobina e glicose plasmática. Contudo, não houve correlação significativa entre os dados biométricos e fisiológicos. Dessa forma, as mudanças ocorridas na fisiologia de Potamotrygon rex sugerem plasticidade da espécie para suportar as variações sazonais da região.

\section{Physiological adaptations of Potamotrygon Rex (Potamotrygonidae) stingles in front of seasonal variations in the environment}

\begin{abstract}
The species of freshwater stingrays (family Potamotrygonidae) have their evolutionary history intrinsically connected to the geological history of the neotropical watersheds. The representatives of this group accumulate a series of physiological adaptations that make it possible to maintain their populations in varying environmental conditions, most of which are still unknown. Thus, this work aimed to analyze the physiological responses of Potamotrygon rex rays, in the face of variations in the environment, which occurred during the hydrological cycle (rainy/dry periods) in the Upper/Middle Tocantins River region. The environment was verified in the taking of water samples for seven consecutive weeks, in the rainy and dry season, in the same region where the rays were collected. The specimens were collected in the transition area of the Lajeado reservoir, on the Tocantins River, from which biometric data were recorded and blood samples were collected to verify the physiological indicators (hematocrit, hemoglobin and plasma glucose). The stingray relative condition factor (Kn) was calculated, and the relationship between biometric data and physiological variables was assessed by Pearson's correlation. As a result, the environmental changes that occur seasonally during the hydrological cycle (rainy and dry periods) were verified. In the rainy season (from October to April), the water temperature $\left({ }^{\circ} \mathrm{C}\right.$ ), the oxygen dissolved in the water $(\mathrm{mg} / \mathrm{L})$ and the turbidity showed higher values than in the dry season (from May to September), with exception of electrical conductivity. For the specimens collected during the dry season, there was an increase in weight, $\mathrm{Kn}$, hematocrit, hemoglobin and plasma glucose. However, there was no significant correlation between biometric and physiological data. Thus, changes in the physiology of Potamotrygon rex suggest plasticity of the species to withstand seasonal variations in the region.
\end{abstract}

Keywords: Potamotrygonidae; Physiological variations; Environment; Seasonality.

Topic: Desenvolvimento, Sustentabilidade e Meio Ambiente

Reviewed anonymously in the process of blind peer.

Juliane Monteiro dos Santos

Universidade Federal do Tocantins, Brasil http://lattes.cnpq.br/9630851085710012

http://orcid.org/0000-0002-3832-5295

julianemonteiro812@gmail.com

Derval Gomes Ribeiro Neto (io

Universidade Federal do Tocantins, Brasil http://lattes.cnpq.br/0793667599069011

http://orcid.org/0000-0003-1031-5009

derval.paladino@gmail.com

Elineide Eugênio Marques (id

Universidade Federal do Tocantins, Brasil

http://lattes.cnpq.br/9875056556045599

http://orcid.org/0000-0003-0223-6853

emarques@mail.uft.edu.br

d.

DOI: 10.6008/CBPC2179-6858.2020.007.0033
Received: 04/12/2020

Approved: $\mathbf{2 1 / 1 2 / 2 0 2 0}$

Carla Simone Seibert (iD

Universidade Federal do Tocantins, Brasi http://lattes.cnpq.br/6679543572745031

http://orcid.org/0000-0002-3988-7767

carlaseibert@yahoo.com
Referencing this:

SANTOS, J. M.; RIBEIRO NETO, D. G.; MARQUES, E. E.; SEIBERT, C. S.. Adaptações fisiológicas de arraias Potamotrygon Rex

(Potamotrygonidae) frente às variações sazonais no ambiente. Revista Ibero Americana de Ciências Ambientais, v.11, n.7, p.409-423, 2020. DOI: http://doi.org/10.6008/CBPC2179-6858.2020.007.0033 


\section{INTRODUÇÃO}

Os ambientes aquáticos tropicais, principalmente os de água doce, como, o rio Tocantins, são bastante diversos e dinâmicos em relação às suas propriedades físicas e químicas (MARQUES, 2006; LOLIS, 2008; BENEVIDES, 2010; PINTO et al., 2019). São, portanto, tipicamente instáveis e caracterizados por marcantes variações naturais nas suas propriedades (TUNDISI et al., 2008; JUNK, 2013).

Dentre os diversos elementos climáticos a precipitação, em seu regime sazonal, é uma das variáveis que exerce maior influência nas características do ambiente aquático (RIBEIRO et al., 1995; BRITO et al., 2006). Entretanto, além da precipitação, outros elementos climatológicos podem variar de acordo com a localidade observada, assim, as variações anuais da temperatura, umidade e a radiação solar também interagem com os sistemas aquáticos, podendo produzir efeitos importantes sobre as comunidades aquáticas e ocasionando modificações nos componentes bióticos e abióticos do sistema (TUNDISI et al., 2008; OLIVER et al., 2014).

Neste contexto, as variações ambientais como mudanças rápidas ou extremas na temperatura, na concentração de oxigênio dissolvido, no pH, na concentração e nos tipos de íons, podem ocasionar diferentes níveis de estresse, e reduzir a habilidade dos organismos em manter a homeostase (BONGA, 1997; MARIANO et al., 2009). Geralmente a resposta dos organismos aos agentes estressores depende do grau e da duração do estímulo. Em regiões tropicais a maioria das espécies de peixes, geralmente estão adaptadas às flutuações ambientais moderadas, apresentando mecanismos compensatórios para minimizar o estresse crônico (ANDRADE-TALMELLI et al., 1999; SIMPLENDORFER et al., 2011; DUNCAN, 2016; SEERS-ROESCH et al., 2012a). Portanto, em reposta ao estresse ambiental os organismos podem procurar novos ambientes, ou desenvolver mudanças teciduais, fisiológicas e comportamentais (MUUSZE et al., 1998; ZENI et al., 2016). E, a partir destas adaptações podem assegurar sua sobrevivência, reprodução, desenvolvimento, distribuição geográfica e até mesmo a diversificação das espécies (RANDALL et al., 2006; ZENI et al., 2016).

No caso da bacia do rio Tocantins existe uma diversidade de ambientes com características particulares, que proporcionam condições para uma fauna de peixes diversificada, que se desenvolveu associada a história evolutiva da bacia (LUCINDA et al., 2007; PINTO et al., 2019). Dentre os grupos presentes há um grupo muito particular de elasmobrânquios, as arraias da família Potamotrygonidae. As espécies que pertencem a esta família derivam de grupos marinhos e apresentam adaptações para viverem exclusivamente em ambiente de água doce, estando distribuídas em quatro gêneros, Potamotrygon, Paratrygon, Plesiotrygon e Heliotrygon (CARVALHO et al., 2004; ROSA et al., 2010; CARVALHO et al., 2011).

A literatura tem evidenciado que, assim como observado em outros elasmobrânquios, os potamotrigonídeos apresentam preferências quanto à ocupação e uso de hábitat associados às condições ambientais (ALMEIDA et al., 2009; DUNCAN et al., 2011; DUNCAN, 2016). Os ajustes fisiológicos ocorridos ao longo do processo evolutivo das arraias de água doce possibilitaram sua adaptação aos filtros ambientais e o estabelecimento da espécie em vários ambientes (HOPKINS et al., 2003; DUNCAN, 2008; OLIVEIRA, 2008; SPEERS-ROESCH et al., 2012; OLIVEIRA, 2013; DUNCAN et al., 2011). 
A espécie Potamotrygon rex é uma arraia que possui distribuição concentrada no alto e médio rio Tocantins (CARVALHO, 2016). A presença dos indivíduos desta espécie é frequentemente observada na região do médio rio Tocantins, especialmente no reservatório da UHE-Lajeado (observação pessoal). Indivíduos desta espécie apresentam coloração dorsal preta a marrom escuro, e, juntamente com as espécies Potamotrygon henlei e Potamotrygon leopoldi formam o grupo de espécies denominado "arraias negras" (CARVALHO, 2016). Contudo, como observado para outros potamotrigonídeos da região Amazônica, os estudos relacionados à Potamotrygon rex são escassos e limitam-se a alguns aspectos relacionados, por exemplo, a sistemática e distribuição (CARVALHO, 2016) e ao veneno (SANTOS et al., 2019). Sendo que, até o presente momento há uma lacuna no entendimento de como a espécie de arraia Potamotrygon rex responde às variações sazonais do ambiente aquático.

Neste sentindo, o estudo dos parâmetros sanguíneos é uma importante ferramenta que permite compreender e avaliar o estado fisiológico dos peixes, podendo ser utilizada, dentre outras coisas, para o diagnóstico de doenças; caracterizar as diferentes estratégias no que se refere à demanda metabólica por oxigênio e investigar estratégias fisiológicas adaptativas relacionadas às variações ambientais (VAL et al., 1998; TAVARES-DIAS et al., 2006; PAVLIDIS et al., 2007; ALMEIDA-VAL et al., 2011; TAVARES-DIAS et al., 2014). Segundo Oliveira (2013), somente a partir de um conhecimento integrado entre a investigação do ambiente e a relação com os distúrbios fisiológicos, que acometem os diversos organismos aquáticos, será possível evoluir na formulação de ações que auxiliem na avaliação e controle de saúde das espécies, contribuindo para seu bem estar.

No que tangue as arraias, Oliveira (2013) destaca a importância de conhecer a fisiologia do sangue das espécies de arraias amazônicas, e ressalta ser esta uma ferramenta adequada para compreender as respostas fisiológicas geradas pelas arraias de água doce, frente à diversidade e intensidade das variações do ambiente, seja pela ação antropológica e/ou natural. Entretanto, mesmo a hematologia de peixes sendo uma ferramenta valiosa, o progresso no estabelecimento de parâmetros hematológicos é lento e ainda pouco usado para espécies em seu ambiente natural (VAL et al., 1998; TAVARES-DIAS et al., 2006). Com relação às arraias três questões dificultam os estudos da fisiologia de espécies de ambiente natural: a dificuldade da coleta, o receio do acidente (devido à manipulação errada) e a ausência de kits bioquímicos específicos para o grupo.

Portanto, os estudos sobre como as arraias se adaptam as mudanças ocorridas no ambiente aquático, de forma sazonal, são relevantes para o conhecimento de estratégias adaptativas fisiológicas, diagnóstico do estado de saúde e no manejo, podendo resultar em cuidados mais eficientes para as espécies nativas como, por exemplo, a Potamotrygon rex. Investigações deste tipo podem, inclusive, auxiliar na criação e manutenção desta espécie em cativeiro, diminuindo assim, o possível impacto da retirada deste grupo de seu ambiente natural, como já vem ocorrendo para outras espécies amazônicas, com intuito de suprir a crescente demanda do mercado, tanto de aquariofilia, quanto do consumo da carne de arraia (ARAÚJO et al., 2004; DUNCAN et al., 2010).

Desse modo, este trabalho visa analisar as respostas fisiológicas da arraia Potamotrygon rex, frente 
as variações do ambiente, ocorridas durante o ciclo hidrológico (períodos chuvoso/estiagem), na região do Alto/Médio rio Tocantins.

\section{MATERIAIS E MÉTODOS}

\section{Caracterização da área de estudo}

As coletas foram realizadas na área do reservatório da UHE Lajeado (ou Luís Eduardo Magalhães), está localizado entre os municípios de Miracema do Tocantins e Brejinho do Nazaré, mais especificamente, na região de Porto Nacional (Figura 1). Neste município está a área de transição do reservatório, à cerca de $1400 \mathrm{~km}$ da foz do rio Tocantins, localizada em Belém, PA.

O reservatório (lago) da UHE Lajeado foi concluído em fevereiro de 2001, quando atingiu sua cota de operação (212 m); tem cerca de $172 \mathrm{Km}$, área superficial de $630 \mathrm{~km}^{2}$, área de drenagem 184.219,00 km², nível a montante de $212 \mathrm{~m}$ e a jusante de $175,80 \mathrm{~m}$. A UHE Lajeado opera a fio d'água e a oscilação do nível está relacionada à operação da própria usina e de outros represamentos localizado a montante (LOLIS, 2008).

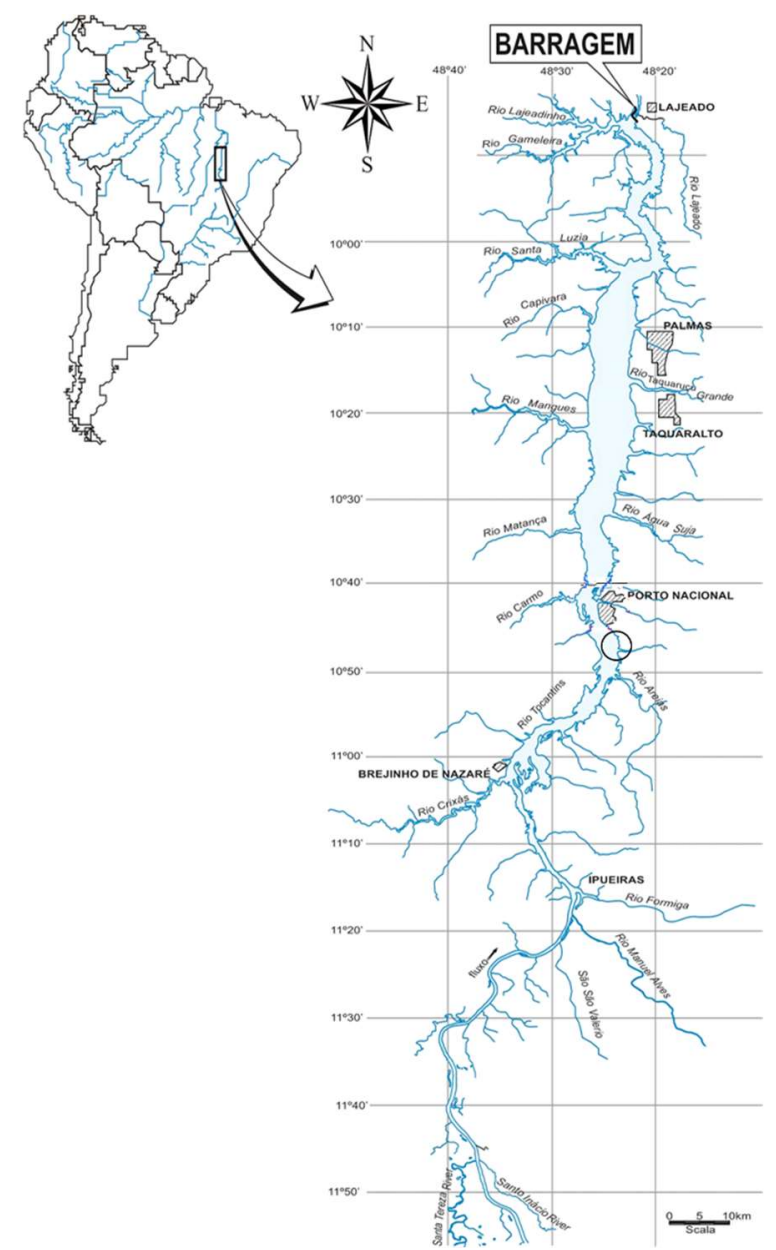

Figura 1: Localização da área de amostragem (indicada pelo círculo), no reservatório da Usina de Lajeado (UHE Luís Eduardo Magalhães), rio Tocantins.

\section{Parâmetros físico-químicos da água}

As amostras foram concentradas no município de Porto Nacional (Tocantins) região a montante da UHE- Lajeado (Figura 1). As coletadas foram realizadas entre janeiro e março de 2018, no período chuvoso. 
No período de estiagem as coletas ocorreram entre junho e agosto 2018. Em ambos os casos, as coletas foram realizadas semanalmente (uma vez na semana). Em cada coleta foram registradas na superfície da água, a temperatura $\left({ }^{\circ} \mathrm{C}\right), \mathrm{pH}$, condutividade $(\mu \mathrm{S} / \mathrm{cm})$ e oxigênio dissolvido $(\mathrm{mg} / \mathrm{L})$, utilizando sondas multiparâmetro, entre 8 e 9 horas da manhã. Amostras de água também foram coletadas e armazenadas em gelo e posteriormente transportadas a laboratório de Microbiologia Ambiental (LAMBIO), da Universidade Federal do Tocantins, para análise da turbidez.

\section{Coleta de sangue e dados biométricos}

As coletas das arraias Potamotrygon rex (Figura 2) foram realizadas no ano de 2018 (Licença do Sisbio/ICMbio $n^{\circ} 45407-/ 2017$ ) e as capturas ocorreram em dois períodos hidrológicos distintos, período chuvoso (entre os meses de janeiro a março) e período de estiagem (entre os meses de junho a agosto). A amostra total correspondeu a 25 animais, sendo 12 (período chuvoso) e 13 (período de estiagem).

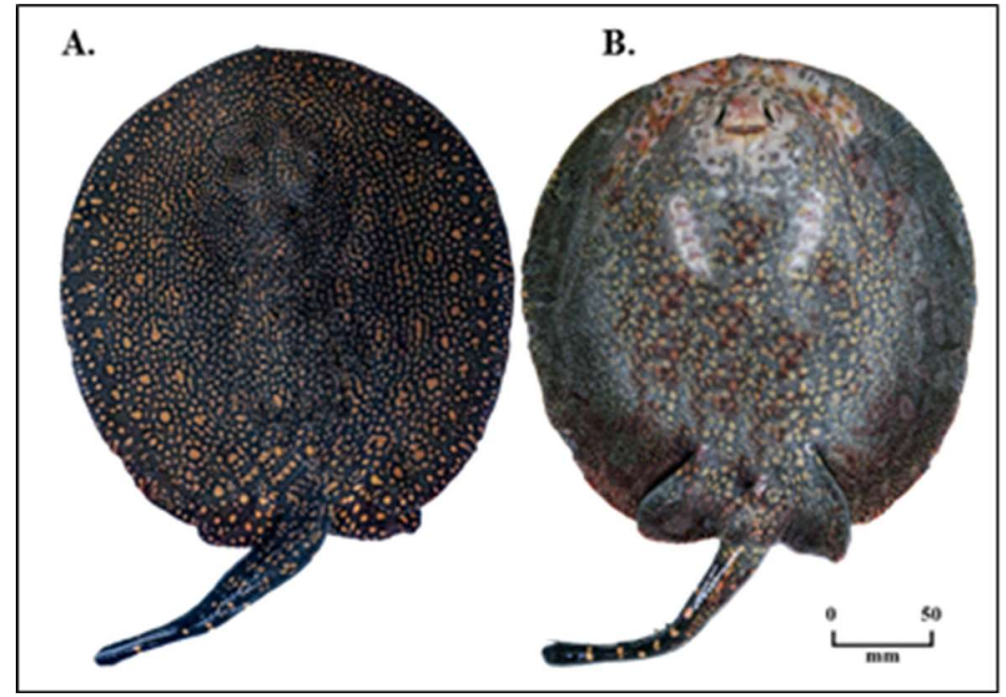

Figura 2: Arraias Potamotrygon rex. Fotografia da fêmea (A) visão dorsal e (B) visão ventral.

Para as coletas foi utilizado uma adaptação do apetrecho de pesca denominado "Nasa", amplamente utilizado na Colômbia e na Venezuela (AJIACO-MARTínEZ et al., 2012). As adaptações realizadas foram o aumento do cabo de sustentação, aumento do diâmetro do cone de ferro, e a diminuição do tamanho da malha do material utilizado para confecção do saco de armazenagem. É possível observar em detalhe o apetrecho e sua utilização nas figuras 3 A e B. Após a coleta, as arraias foram transferidas para um viveiro montado no ambiente onde ocorreram as capturas (Figura $3 \mathrm{C}$ ), no qual permaneceram por um período máximo de 12 horas, com o objetivo de minimizar o estresse associado à coleta. Posteriormente, os espécimes foram retirados cuidadosamente do viveiro, com um puçá de mão e colocados em caixas plásticas com anestésico (Eugenol 0,2 g/L; Figura $3 \mathrm{D}$ ) para a coleta de sangue.

As coletas de sangue ocorreram entre 8 e $9 \mathrm{~h}$, nos mesmos horários em que foram realizadas as coletas dos parâmetros ambientais. Os procedimentos de manuseio e coleta sanguínea seguiram as recomendações de Oliveira (2013), que consiste na punção do vaso branquial. As amostras de sangue foram colocadas em dois tubos diferentes: um com ácido etilenodiamino tetra-acético a 10\% (EDTA), para 
determinação da hemoglobina e hematócrito, e outro com fluoreto de sódio, para dosagem de glicose. O hematócrito foi determinado pela técnica de microhematócrito e a concentração de hemoglobina plasmática pela técnica de cianometahemoglobina (LEWIS et al., 2005). A determinação da concentração de glicose foi realizada por métodos enzimático-colorimétrico quantificado pelo kit comercial Glicose Vet da Biotécnica.

Após a coleta de sangue, foi realizada a coleta dos dados biométricos: (i) comprimento do disco, (ii) largura do disco e (iii) peso total, seguindo a metodologia descrita por Rosa (1985) e Carvalho (2016).

Para obtenção dos valores esperados foi utilizado inicialmente a relação peso-comprimento $\mathrm{Wt}=\mathrm{aLd}^{\mathrm{b}}$ (Wt - peso total em gramas; Ld - largura do disco em cm, $a$ e $b$ são constantes). Esses valores foram utilizados para calcular o fator de condição relativo $(\mathrm{Kn}=\mathrm{Wt} / \mathrm{We})$, através do quociente entre o peso registrado $(\mathrm{Wt})$ e o peso esperado (We) para um dado comprimento (LE CREN, 1951). Para as arraias foi considerado a largura do disco (Ld), seguindo Araújo (1998). Após estimativa dos valores individuais de Kn de todos as arraias, foi calculado o valor médio de Kn dos exemplares agrupados para os dois períodos, chuvoso e estiagem.

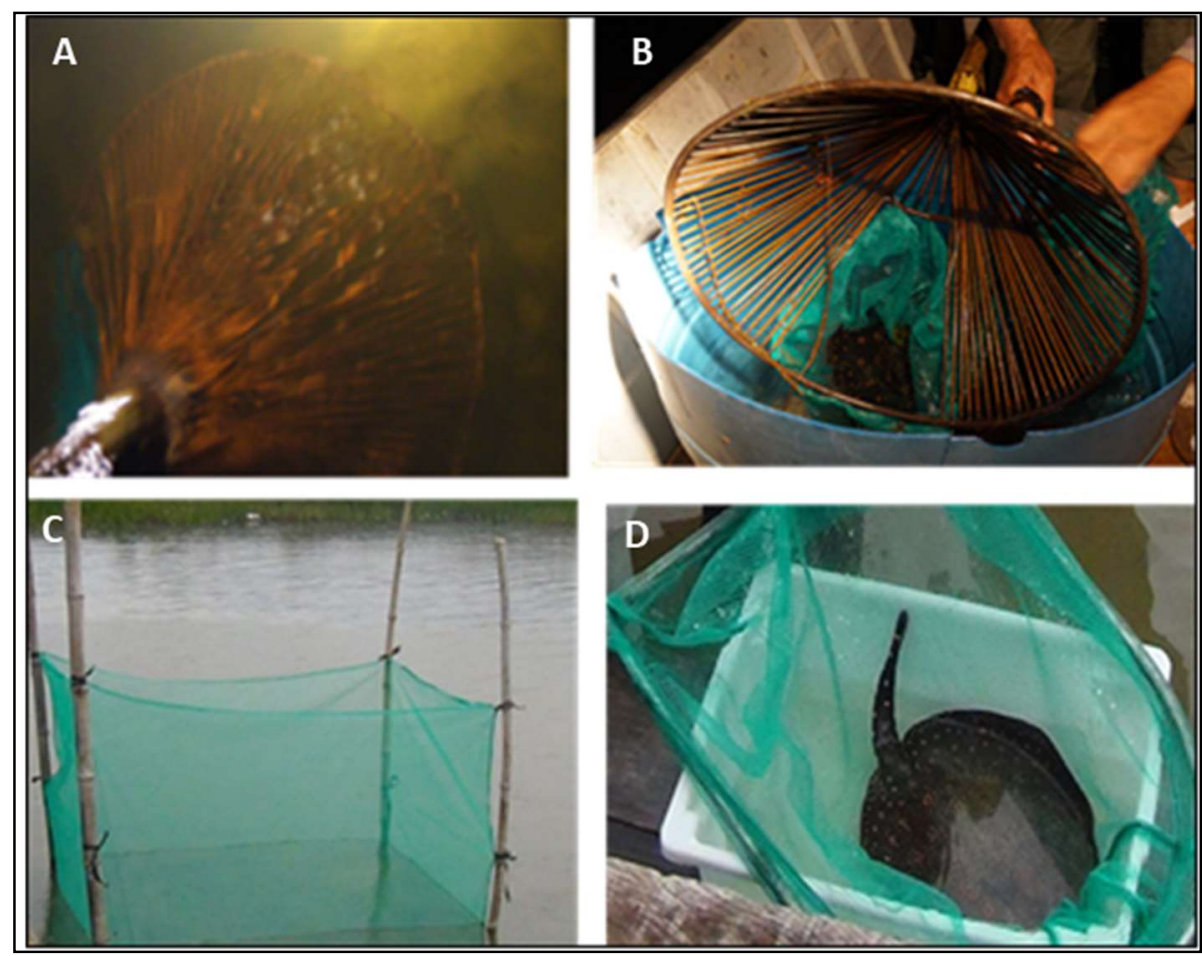

Figura 3: Apetrecho utilizado para coleta de arraia Potamotrygon rex - (A) Operação do apetrecho de coleta; (B) detalhe da retirada do animal capturado do aparelho de coleta; (C) detalhe do viveiro utilizado para não estressar o animal, e (D) detalhe da arraia sendo anestesiada.

\section{Análises estatísticas}

Para averiguar a normalidade dos dados foi utilizado o teste de normalidade de Shapiro-Wilk (SHAPIRO et al., 1965). Posteriormente foi aplicado o teste $t$ de Student, para comparar e identificar diferença estatisticamente significativa entre os períodos (chuvoso e estiagem). A correlação entre dados biométricos e os indicadores fisiológicos (hematócrito, hemoglobina e glicose) foi realizada com o teste estatístico de correlação de Pearson, usando o Software PAST (PAleontological STatistics) versão 3.24. Os resultados foram expressos em média e desvio padrão (DP), sendo os testes significativos para $p<0,05$. 


\section{RESULTADOS}

\section{Características Ambientais}

A comparação entre as variáveis ambientais foi significativamente diferente entre o período chuvoso e a estiagem. Na estiagem, a temperatura da água, o oxigênio dissolvido e a turbidez foram menores do que no período chuvoso. Em contraposição, os valores da temperatura do ar e da condutividade elétrica foram maiores na estiagem. Esses resultados indicam que as condições do ambiente variam sazonalmente (Tabela 1).

No período chuvoso aumenta o volume e a turbidez da água, a ocorrência de ventos (banzeiro) e de chuvas, que dificultam a visualização e captura dos animais. Contudo, no período de estiagem ocorre o inverso. Há redução na turbidez (aumento na transparência), ausência de chuva e ocorrência esporádica de vento, o que facilita a visualização e captura dos animais, que podem ser observados próximos às margens com frequência.

Tabela 1: Variáveis ambientais no reservatório UHE- Lajeado (UHE Luís Eduardo Magalhães; médio Rio Tocantins), durante o período chuvoso e de estiagem. Dados apresentados como média \pm desvio padrão; valores mínimos máximos, entre parênteses.

\begin{tabular}{|c|c|c|c|}
\hline \multirow[b]{2}{*}{ Variáveis Ambientais } & \multicolumn{2}{|l|}{ Período } & \multirow[b]{2}{*}{$p$-valor } \\
\hline & $\begin{array}{l}\text { Chuvoso } \\
\text { (min-máx) }\end{array}$ & $\begin{array}{l}\text { Estiagem } \\
\text { (min-máx) }\end{array}$ & \\
\hline Temperatura do $\operatorname{ar}\left({ }^{\circ} \mathrm{C}\right)$ & $\begin{array}{l}26,88 \pm 0,84 \\
(25,96-28,38)\end{array}$ & $\begin{array}{l}30,48 \pm 0,51 \\
(29,84-31,28)\end{array}$ & 0,0005 \\
\hline Temperatura da água $\left({ }^{\circ} \mathrm{C}\right)$ & $\begin{array}{l}29,50 \pm 0,77 \\
(28,4-31,0)\end{array}$ & $\begin{array}{l}25,98 \pm 0,81 \\
(24,4-27,4)\end{array}$ & 0,0009 \\
\hline Potencial hidrogênico $(\mathrm{pH})$ & $\begin{array}{l}7,98 \pm 0,54 \\
(7,75-8,06)\end{array}$ & $\begin{array}{l}7,71 \pm 0,40 \\
(7,03-8,18)\end{array}$ & 0,1812 \\
\hline $\begin{array}{l}\text { Oxigênio Dissolvido (OD, } \\
\mathrm{mg} / \mathrm{L})\end{array}$ & $\begin{array}{l}7,02 \pm 0,50 \\
(6,81-7,16)\end{array}$ & $\begin{array}{l}5,01 \pm 0,73 \\
(4,85-5,12)\end{array}$ & 0,0004 \\
\hline $\begin{array}{l}\text { Condutividade elétrica } \\
(\mu \mathrm{S} / \mathrm{cm})\end{array}$ & $\begin{array}{l}56,84 \pm 2,10 \\
(53,4-63,9)\end{array}$ & $\begin{array}{l}78,71 \pm 4,0 \\
(73,6-84,4)\end{array}$ & 0,0001 \\
\hline Turbidez da água (NTU) & $\begin{array}{l}5,30 \pm 1,8 \\
(3,75-6,78)\end{array}$ & $\begin{array}{l}1,59 \pm 0,4 \\
(0,94-2,08)\end{array}$ & 0,0006 \\
\hline
\end{tabular}

\section{Parâmetros biométricos}

Os valores médios das medidas biométricas de Potamotrygon rex foram semelhantes entre os períodos chuvoso e de estiagem. As diferenças significativas foram observadas somente para o peso dos indivíduos (Tabela 2).

A equação da relação entre o peso total e a largura do disco para todos os indivíduos agrupados foi a seguinte: $\mathrm{Wt}=0,0002 \mathrm{Ld}^{4,161}$, com $\mathrm{R}^{2}=0,6852$. Os valores do fator de condição relativo $(\mathrm{Kn})$ mostraram que as arraias coletadas no período de estiagem apresentaram diferença entre as estações, sendo o valor médio do Kn significativamente maior na estiagem ( $p=0,0039)$ (Figura 4).

Tabela 2: Medidas biométricas e fator de condição da arraia de água doce Potamotrygon rex. Dados apresentados como média \pm desvio padrão; valores mínimos - máximos, entre parênteses.

\begin{tabular}{llll}
\hline \multirow{2}{*}{ Parâmetros Biométricos } & Período & p-valor \\
\cline { 2 - 3 } & $\begin{array}{l}\text { Chuvoso } \\
\text { (min-máx) }\end{array}$ & $\begin{array}{l}\text { Estiagem } \\
\text { (min-máx) }\end{array}$ & 0,1456 \\
\hline Comprimento do disco $(\mathrm{cm})$ & $\begin{array}{l}38,91 \pm 4,19 \\
(34,1-47,7)\end{array}$ & $\begin{array}{l}40,92 \pm 3,04 \\
(35,0-47,0)\end{array}$ & \multirow{2}{*}{ (3) }
\end{tabular}


Largura do disco $(\mathrm{cm})$

Peso (g)
$36,73 \pm 4,06$

$(32,1-45,2)$

$458,97 \pm 186,01$

$(306,17-462,06)$
$40,07 \pm 2,54$

$(37,0-44,8)$

$882,07 \pm 221,52$

$(868,63-1735,27)$
0,1554

0,0003

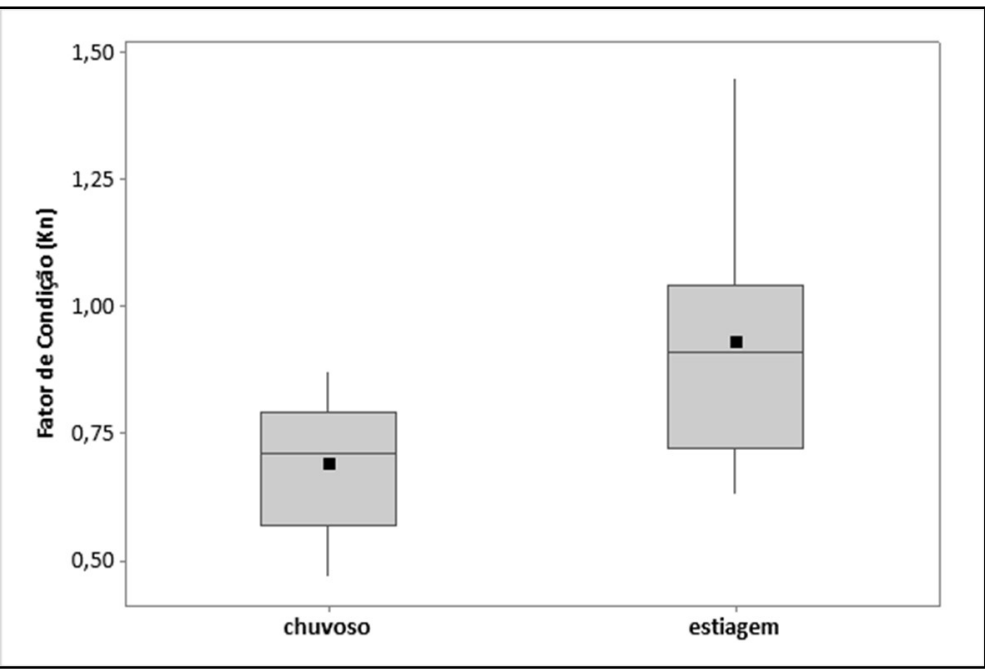

Figura 4: Valores médios, desvio padrão e amplitude de variação do fator de condição relativo (Kn) de Potamotrygon rex em relação ao período chuvoso e de estiagem.

\section{Parâmetros Hematológicos (hematócrito, concentração plasmática de hemoglobina e glicose plasmática)}

Para os indicadores fisiológicos de Potamotrygon rex, os espécimes coletados no período de estiagem apresentaram os maiores percentuais de hematócrito, de concentração de hemoglobina e de glicose plasmática (Tabela 3). A hemoglobina plasmática foi, em média, 59\% maior no período de estiagem e a glicose plasmática 319\% (Tabela 3). Ao correlacionar os valores biométricos com os indicadores fisiológicos (hematócrito, hemoglobina e glicose) da arraia Potamotrygon rex, observou-se que não houve correlação significativa entre os dados.

Tabela 3: Hematócrito, concentração de hemoglobina plasmática e glicose plasmática de Potamotrygon rex no período chuvoso e de estiagem. Dados apresentados como média \pm desvio padrão.

\begin{tabular}{|c|c|c|c|}
\hline \multirow[b]{2}{*}{ Variáveis sanguíneas } & \multicolumn{2}{|l|}{ Período } & \multirow[b]{2}{*}{$p$-valor } \\
\hline & $\begin{array}{l}\text { Chuvoso }(\mathrm{N}=12) \\
\text { (min-máx) }\end{array}$ & $\begin{array}{l}\text { Estiagem }(\mathrm{N}=13) \\
(\mathrm{min}-\mathrm{máx})\end{array}$ & \\
\hline Hematócrito (\%) & $\begin{array}{l}20,67 \pm 1,72 \\
(17-23)\end{array}$ & $\begin{array}{l}22,54 \pm 1,20 \\
(20-25)\end{array}$ & 0.004 \\
\hline Hemoglobina plasmática $(\mathrm{g} / \mathrm{dL})$ & $\begin{array}{l}3,57 \pm 0,47 \\
(2,97-3,89)\end{array}$ & $\begin{array}{l}\mathbf{6 , 0 2} \pm 0,70 \\
(5,2-7,2)\end{array}$ & 0,001 \\
\hline Glicose plasmática (mg/dL) & $\begin{array}{l}38,81 \pm 5,54 \\
(32,7-48,5)\end{array}$ & $\begin{array}{l}\mathbf{1 2 1 , 5} \pm \mathbf{3 , 2} \\
(101-133,1)\end{array}$ & 0,0006 \\
\hline
\end{tabular}

\section{DISCUSSÃO}

As características do ambiente aquático podem variar em função das características dos corpos de água, da intensidade da radiação solar, intensidade pluviométrica, ação do vento e de outras forças que atuam no ambiente aquático (BRITTO et al., 2006; TUNDISI et al., 2008; JUNK, 2013).

$\mathrm{Na}$ área de estudo, inserida no alto médio rio Tocantins, a ocorrência de período chuvoso (outubro a abril) e de estiagem (maio a setembro) bem definidos durante o ano, contribuem para uma sazonalidade marcante no ambiente aquático (LOLIS, 2008; MARCUZZO et al., 2013; PINTO, 2013; PINTO et al., 2019; SOUZA, 2016; ROLDÃO et al., 2019). A ocorrência de chuvas é um dos fatores que controla o aporte de 
nutrientes em rios, lagos e reservatórios e, consequentemente, influencia no comportamento hidrodinâmico do corpo d'água, principalmente nos mecanismos de transporte, ou seja, turbulência, difusão, dispersão, entre outros (TUNDISI et al., 2008; OLIVER et al., 2014).

Neste sentido, a ocorrência de chuvas proporciona o carreamento de nutrientes do entorno da bacia para dentro do ambiente aquático no período chuvoso, quando a temperatura do ar e da água geralmente são mais elevadas. Ao mesmo tempo o aumento da turbidez restringe a penetração da radiação solar no ambiente aquático, sendo as contribuições alóctones importantes, especialmente nas regiões próximas às margens, onde ocorre também os bancos de macrófitas (MARQUES, 2006; LOLIS, 2008; BENEVIDES, 2010).

Por outro lado, no período de estiagem, a redução da turbidez da água possibilita a entrada da radiação solar até profundidades maiores. Assim, ocorre a intensificação dos processos metabólicos no ambiente (processos autóctones), consumindo a matéria orgânica carreada pelas chuvas e/ou produzidas no próprio ambiente, conforme indicada pelo aumento da condutividade da água e redução do oxigênio dissolvido neste período (Tabela 1). A redução na turbidez da água (aumento da transparência) é um filtro ambiental importante em ambientes de reservatório influenciando os processos seletivos e adaptativos de toda a fauna aquática (PINTO et al., 2019).

Neste contexto, dentre os gases dissolvidos na água, o oxigênio é um dos mais relevantes na dinâmica e caracterização dos ecossistemas aquáticos (ESTEVES, 2011). Neste ambiente alguns fatores, como por exemplo, a decomposição da matéria orgânica, perdas para respiração de organismos aquáticos e aumento da temperatura auxiliam na redução do oxigênio dissolvido na água (TAVARES-DIAS et al., 2006; CHIPPARIGOMES et al., 2005). Além disso, a elevada atividade biológica em altas temperaturas tende a reduzir a concentração de oxigênio, principalmente no período noturno, quando não há a contribuição da produção pela fase luminosa da fotossíntese (ESTEVES, 2011). Dessa forma, em altas temperaturas os peixes necessitam de mais oxigênio, embora este tende a se encontrar menos disponível no ambiente (BALDISSEROTTO, 2009).

Mudanças na concentração de oxigênio, do ambiente, pode ocasionar efeitos na fisiologia, comportamento e na distribuição de espécies, especialmente em regiões amazônicas, onde existem baixas tensões de oxigenação em altas temperaturas (VAL et al., 1992; VAL et al., 1998; TAVARES-DIAS et al., 2006; PAVLIDIS et al., 2007; TAVARES-DIAS et al., 2014). Por esta razão, algumas espécies desenvolveram mecanismos adaptativos ao longo de sua história evolutiva para viver em ambientes com baixa oxigenação, desenvolvendo ajustes fisiológicos (RANDALL et al., 2006) bioquímicos, teciduais e estratégia comportamentais (MUUSZE et al., 1998). Na literatura há indícios de que as arraias de água doce, ao logo do processo evolutivo, passaram por ajustes fisiológicos que possibilitaram sua adaptação aos filtros ambientais, o que possibilitou seu estabelecimento nos mais variados ambiente (HOPKINS et al., 2003; DUNCAN, 2008; OLIVEIRA, 2008; SPEERS-ROESCH et al., 2012; OLIVEIRA, 2013).

Nesse estudo, foi verificado o aumento do peso total (Tabela 2) e melhora do fator de condição para Potamotrygon rex, no período de estiagem (Figura 4). E estes resultados podem estar relacionados a dois fatores principais, a alimentação e/ou reprodução. O primeiro reforça que os resultados relacionadas as 
diferentes na dieta das arraias de água doce podem ter relação com às oscilações do nível hidrométrico e da disponibilidade dos recursos alimentares, como verificado por Speers-Roesch et al. (2010) em elasmobrânquios, ou resultam de mudanças ontogenéticas e espaço-temporais caracterizando uma flexibilidade trófica das espécies (CHARVET-ALMEIDA, 2006; RINCON, 2006; ALMEIDA et al., 2009; SHIBUYA et al., 2010; GARRONE NETO et al., 2012; PAGLIARINI et al., 2020).

No caso de potamotrigonideos, que se alimentam no fundo, geralmente próximo as regiões litorâneas onde são observados com frequência, a qualidade e/ou qualidade do alimento disponível no período de estiagem pode favorecer esses animais. Neste período, o aumento da condutividade, da disponibilidade de fósforo e de nitrogênio, provavelmente devido ao aumento dos processos de decomposição e recirculação de nutrientes, pode modificar a quantidade e/ou qualidade do alimento ingerido. Ao mesmo tempo, mudanças nos mecanismos de deteç̧ão e captura de presas, devido as condições apresentadas ao longo do ano no ambiente aquático, podem favorecer a captura dos alimentos (BATISTA, 2008; ALMEIDA et al., 2009; SHIBUYA et al., 2010).

Neste sentindo, Lonardoni et al. (2006) observaram variação sazonal na qualidade e frequência de ocorrência dos recursos alimentares entre o período de seca e cheia no alto rio Paraná, para Potamotrygon falkneri e Potamotrygon motoro, o que ainda segundo os autores, demonstra que o ambiente também pode ser um fator determinante nas diferentes estratégias adotadas pelas arraias.

Vasconcelos et al. (2011) também encontraram diferença sazonal na dieta de Potamotrygon motoro, composta principalmente de insetos no período de seca e com predominância de molusco, crustáceo e peixes no período de cheia. Para Pagliarini et al. (2020), há correlação entre o aumento da proteína muscular das arraias de água doce e em relação aos itens alimentares, principalmente macroinvertebrados bentônicos.

Com relação ao fator reprodutivo o incremento no peso estaria ligado ao processo de armazenamento de nutrientes no fígado, antes do início do período reprodutivo (ARAÚJO, 1998). Entretanto, como os animais coletados não estavam em reprodução, sendo provavelmente todos juvenis (comprimento do disco menor ou igual $47,7 \mathrm{~cm}$ ), há necessidade de investigações direcionadas para se inferir sobre os parâmetros reprodutivos, considerando também a lacuna de informações a este respeito.

O aumento dos indicadores fisiológicos (hematócrito, hemoglobina e glicose plasmática) na estiagem (Tabela 3), por sua vez, sugerem a condição de estresse ambiental, gerado pelas oscilações dos parâmetros físicos-químicos do ambiente. Assim, as variações fisiológicas dos osteíctes e elasmobrânquios em respostas a variação das características ambientais da água, como por exemplo, mudanças de temperatura, na concentração de oxigênio dissolvido, no pH, na concentração e nos tipos de íons, são desencadeadas de forma que o indivíduo busque sempre o retorno da homeostase, por meio de ajustes e adaptações fisiológicas (BONGA, 1997; MARIANO et al., 2009; OLIVEIRA, 2013; DUNCAN, 2016).

Algumas espécies da região amazônica tanto de peixes (VAL et al., 1998; TAVARES-DIAS et al., 2006) como de potamotrigonídeos (DUNCAN, 2008; OLIVEIRA, 2008; OLIVEIRA, 2013; DUNCAN et al., 2011) são bem adaptadas as variações sazonais do ambiente. Assim, o sucesso das espécies depende dos ajustes fisiológicos desencadeados em condições estressantes para esses animais (RANDALL et al., 2006; ZENI et al., 
2016). Neste contexto, alguns autores, apontam que o estresse induz mudanças hematológicas, seja, pela hemodiluição ou hemoconcentração, alterando os valores de hematócrito e outros parâmetros hematológicos (MCDONALD et al., 1997; TAVARES-DIAS et al., 2006; ALURU et al., 2009). Desse modo, a elevação do hematócrito e da hemoglobina plasmática, observados para arraia Potamotrygon rex, na estiagem, pode ser compreendido como uma estratégia para lidar com a redução do oxigênio da água, observado para esse período (Tabela 1). Para Martin et al. (1978) existem modificações nas hemoglobinas das arraias de água doce da região amazônica que thes possibilitam viver em ambientes com baixa disponibilidade de oxigênio. Para Oliveira (2013), a elevação da hemoglobina apresentada pelas arraias de água doce da região amazônica pode ser entendida como uma resposta fisiológica da espécie, na tentativa de obter uma oxigenação mais eficiente, num período em que há necessidade de elevar e/ou manter a atividade metabólica, como relatado para outros grupos (VAL et al., 1992; TAVARES-DIAS et al., 2006; ALMEIDA-VAL et al., 2011). De maneira geral, os aspectos descritos no presente trabalho apresentaram resultados similares aos encontrados por Oliveira (2008), Oliveira (2013), Duncan et al. (2008), Brinn et al. (2012), Brito (2012), Lemos (2011), Duncan (2016) quando investigaram várias espécies da família Potamotrygonidae.

O aumento da glicose reforça a ideia da existência de estímulos ambientais estressores durante a estiagem. Barcelos et al. (2010), discorrem que o aumento da glicose plasmática aponta que os organismos estiveram submetidos a uma situação possivelmente estressante, mostrando uma tendência à mobilização de reservas energéticas. A mobilização da glicose ocorre como meio para fornecer energia extra ao animal, para que este possa superar o distúrbio imposto (BONGA, 1997). Neste sentido, Pottinger et al. (1995) considera que se ocorrer uma fonte de estresse de natureza respiratória, essas alterações envolveram o aumento da dopamina, essa por sua vez, aumentará os níveis de adrenalina e noradrenalina no plasma, a elevação da concentração de hemoglobina no sangue e de glicose no plasma.

Neste contexto, as respostas fisiológicas apresentadas pela arraia Potamotrygon rex vai ao encontro do que é apresentado para outras espécies de peixes e potamotrigonídeos presentes na região tropical, sendo estes, geralmente bem adaptadas às flutuações ambientais moderadas, apresentando mecanismos compensatórios para minimizar o estresse ocorrido sazonalmente (ANDRADE-TALMELLI et al., 1999; SIMPLENDORFER et al., 2011; CARVALHO et al., 2011; DUNCAN et al., 2011; DUNCAN, 2016).

Por fim, os resultados obtidos para Potamotrygon rex sugerem que a espécie apresenta uma resposta fisiológica às variações sazonais do ambiente, percebidas por meio das variações significativas da hemoglobina, hematócrito e glicose. Este fato abre uma nova perspectiva para o entendimento das relações ecológicas que envolvem a regulação das populações de potamotrigonídeos em seu ambiente natural, para a elaboração de medidas para sua conservação e uso sustentável, bem como, da prospecção dos mecanismos fisiológicos de adaptação deste grupo.

\section{CONCLUSÕES}

O presente trabalho versa sobre os aspectos fisiológicos relacionados a adaptação das arraias de 
água doce Potamotrygon rex frente às alterações sazonais do ambiente aquático (temperatura da água, pH, oxigênio, condutividade, turbidez). Houve aumento do hematócrito, concentração de hemoglobina e glicose plasmática, das arraias no período de estiagem, em resposta à redução de oxigênio e turbidez, e essa plasticidade possibilita a espécie suportar as variações sazonais da região. Entretanto, apesar dos resultados obtidos, o entendimento do comportamento fisiológico desta, e de outras espécies desta família, ainda precisa ser investigado, especialmente em ambiente natural.

AGRADECIMENTOS: À Secretaria Estadual da Educação, Juventude e Esportes do estado do Tocantins (SEDUC-TO) pela concessão da licença para aprimoramento profissional. À CAPES, Edital PROCAD 2013 projeto Fortalecimento de programas de Pós-Graduação, na Amazônia e na Extra-Amazônia, com ênfase em envenenamentos ofídicos: uma estratégia de formação de pessoal e interdisciplinaridade, pelo apoio financeiro. Ao Dr. Rafael de Oliveira, pelas contribuições na análise estatística.

\section{REFERÊNCIAS}

ANDRADE-TALMELLI, E. F.; FENERICH-VERANI, N.; VERANI, J. R.. Fator de condição relativa $(\mathrm{Kn})$ : um critério para selecionar a reprodução de piabanha, Brycon insignis, para indução reprodutiva. Bol. Inst. Pesca, São Paulo, v.25, n.1, p.95-99, 1999.

ALMEIDA, M. P.; BARTHEM, R. B.; VIANA, A. S.; CHARVETALMEIDA, P.. Factors affecting the distribution and abundance of freshwater stingrays (Chondrichthyes: Potamotrygonidae) at Marajó Island, mouth of the Amazon River. Pan-American Journal of Aquatic Sciences, Washington, v.4, n.1, p.1-11, 2009.

ALMEIDA-VAL, V. M. F.; OLIVEIRA, A. R.; SILVA, M. N. P.; FERREIRA-NOZAWA, M. S.; ARAÚJO, R. M.; VAL, A. L.; NOZAWA, S. R.. Anoxia- and hypoxia-induced expression of LDH-A* in the Amazon Oscar, Astronotus crassipinis. Genet. Mol. Biol., São Paulo, v.34, n.2, p.315-

322, 2011. DOI: https://doi.org/10.1590/S1415 $\underline{47572011000200025}$

ALURU, N.; VIJAYAN, M. M.. Stress transcriptomics in fish: A role for genomic cortisol signaling. General and Comparative Endocrinology, Amsterdam, v.164, n.2-3, p.142-150, 2009. DOI: https://doi.org/10.1016/i.ygcen.2009.03.020

AJIACO-MARTÍNEZ, R. E.; RAMÍREZ-GIL, H.; SÁNCHEZDUARTE P.; LASSO C. A.; TRUJILLO, F.. IV. Diagnóstico de la pesca ornamental en Colombia. Bogotá: ARFO Editores e Impressoras Ltda, 2012.

ARAÚJO, M. L. G.. Biologia Reprodutiva e pesca de Potamotrygon sp. (Chondrichthyes-Potamotrygonidae) no médio Rio Negro, Amazonas. Dissertação (Mestrado em Biologia Tropical e Recursos Naturais) - Instituto Nacional de Pesquisa da Amazônia, Manaus, 1998.

ARAÚJO, M. L. G.; CHARVET-ALMEIDA, P.; ALMEIDA, M. P.; PEREIRA, H.. Freshwater stingrays (Potamotrygonidae): status, conservation and management challenges: Information document. AC, 2004.
BALDISSEROTTO, B.. Fisiologia de peixes aplicada à piscicultura. 2 ed. Santa Maria: UFSM, 2009.

BATISTA, W. S.. Caracterização tecnológica e perfil de ácidos graxos em arraias de água doce. Dissertação (Mestrado em Ciência de Alimentos) - Universidade Federal do Amazonas, Manaus, 2008.

BENEVIDES, R. F.. Efeitos do represamento sobre a qualidade da água: o caso da Usina Hidrelétrica Luís Eduardo Magalhães, Tocantins, Brasil. Dissertação (Mestrado em Ciências do Ambiente) - Universidade Federal do Tocantins, Palmas, 2010.

BONGA, S. E. W.. The stress response in fish. Physiol Rev., Washington, v.77, n.3, p.591-625, 1997. DOI: http://doi.org/10.1152/physrev.1997.77.3

BRITO, L. T. L.; SILVA, A. S.; SRINIVASAN, V. S.; GALVÃO, C. O.; GHEYI, H. R.. Uso de análise multivariada na classificação das fontes hídricas subterrâneas da bacia hidrográfica do Salitre. Eng. Agríc., Jaboticabal, v.26, n.1, p.3644, 2006. DOI: https://doi.org/10.1590/S0100$\underline{69162006000100005}$

BRITO, F. M. M.. Variáveis hematológicas, hormonais, bioquímicas, séricas e fauna parasitária em quatro espécies de raias do gênero Potamotrygon Garman, 1877 (Myliobatiformes, Potamotrygonidae) de vida livre. Tese (Doutorado em Patologia Veterinária) - Universidade Estadual Paulista Júlio de Mesquita Filho, Jaboticabal, 2012.

BRINN, R.; MARCON, J.; MCCOMB, D.; GOMES, L.; ABREU, J.; BALDISSEROTO, B.. Stress responses of the endemic freshwater cururu stingray (Potamotrygon cf. histrix) during transportation in the Amazon region of the Rio Negro. Comparative Biochemistry and Physiology, Part A, London, v.162, n.2, p.139-145, 2012. DOI:

https://doi.org/10.1016/j.cbpa.2011.07.004

CARVALHO, M. R.; MAISEY, J. G.; GRANDE, L.. Freshwater stingrays of the Green River formation of Wyoming (Early 
Eocene) with the description of a new genus and species and analysis of its phylogenetic relationships (Chondrichthyes: Myliobatiformes). Bull. Amer. Mus. Nat. Hist., New York, v.284, n.1, p.136-143, 2004.

CARVALHO, M. R.; LOVEJOY, N. R.. Morphology and phylogenetic relationships of a remarkable new genus and two new species of Neotropical freshwater stingrays from the Amazon basin (Chondrichthyes: Potamotrygonidae). Zootaxa, Wellington, v.2776, n.1, p.13-48, 2011.

CARVALHO, M. R.. Potamotrygon rex, a new species of Neotropical freshwater stingray (Chondrichthyes: Potamotrygonidae) from the middle and upper rio Tocantins, Brazil, closely allied to Potamotrygon henlei (Castelnau, 1855). Zootaxa, Wellington, v.4150, n.5, p.537565, 2016

DOI: http://dx.doi.org/10.11646/zootaxa.4150.5.2

CHARVET-ALMEIDA, P.. História natural e conservação das raias de água doce (Chondrichthyes: Potamotrygonidae) no médio rio Xingu, área de influência do projeto hidrelétrico de Belo Monte (Pará, Brasil). Tese (Doutorado em Ciências Biológicas) - Universidade Federal da Paraíba, João Pessoa, 2006.

CHIPPARI-GOMES. A. R.; GOMES, L. C.; LOPES, N. P.; VAL, A. L.; ALMEIDA-VAL, V. M.. Metabolic adjustments in two Amazonian cichlids exposed to hypoxia and anoxia. Comparative Biochemistry and physiology. Biochemistry \& Molecular Biology, Amsterdam, v.141, n.3, p.347-355, 2005 DOI: https://doi.org/10.1016/j.cbpc.2005.04.006

DUNCAN, W. L. P.. Habitat, morfologia branquial e osmorregulação das arraias de água doce da bacia Amazônica (Elasmobranchii: Potamotrygonidae). Tese (Doutorado em Ecologia e Recursos Naturais) - Universidade Federal de São Carlos, São Paulo, 2008.

DUNCAN, W. P.; INOMATA, S. O.; FERNANDES, N. M. Comércio de Arraias de água doce na Região do médio rio Negro, estado do Amazonas, Brasil. Revista Brasileira de Engenharia de Pesca, Rio Grande, v.5, n.2, p.13-22, 2010.

DUNCAN, W.. Ecofisiologia das raias de água doce (Potamotrygonidae): mecanismos de interação organismoambiente. In: LASSO C. A.; ROSA, R.; MORALESBETANCOURT, M. A.; GARRONE NETO, D.; CARVALHO, R. C.. Rayas de agua dulce (Potamotrygonidae) de Suramérica Parte II: Colombia, Brasil, Perú, Bolivia, Paraguay, Uruguay y Argentina. Bogotá: Instituto de Investigación de Recursos Biológicos Alexander von Humboldt, 2016. p.45-64.

DUNCAN, W. P.; FERNANDES, M. N.. Physicochemical characterization of the white, black, and clearwater rivers of the Amazon Basin and its implications on the distribution of freshwater stingrays (Chondrichthyes, Potamotrygonidae). Pan-American Journal of Aquatic Sciences, Washington, v.5, n.3, p.1-11, 2011.

ESTEVES, F. A.. Fundamentos de Limnologia. 3 ed. Engenho Novo, Rio de Janeiro: Interciência, 2011.

GARRONE NETO, D.; UIEDA V. S.. Activity and habitat use of two species of stingrays (Myliobatiformes:

Potamotrygonidae) in the upper Paraná River basin, Southeastern Brazil. Neotropical Ichthyology, Porto Alegre, v.10, n.1, p.81-88, 2012. DOI:

http://dx.doi.org/10.1590/S1679-62252012000100008

HOPKINS, T. E.; CECH, J. J.. The Influence of Environmental Variables on the Distribution and Abundance of Three Elasmobranchs in Tomales Bay, Califórnia. Environmental Biology of Fishes, Corvallis, v.66, p.279-291, 2003. DOI: https://doi.org/10.1023/A:1023907121605

JUNK, J. W.. Current state of knowledge regarding South America wetlands and their future under global climate change. Aquatic Sciences, Zürich, v.75, n.1, p.113-131, 2013.

LEMOS, J. R. G.. Índices parasitários e parâmetros fisiológicos de arraias cururu (Potamotrygon cf. histrix) exportadas como peixes ornamentais: ferramentas para avaliação do estado de saúde da espécie. Dissertação (Mestrado em Diversidade Biológica) - Universidade Federal do Amazonas, Manaus, 2011.

LE CREN, E. D.. The length-weight relationship and seasonal cycle in gonadal weight condition in the perch Perca fluviatilis. Jornal of Animal Ecology, Berlin, v.20, n.2, p.201219, 1951.

LEWIS, S. M.; BAIN, B. J.; BATES, I.. Hematologia prática de Dacie e Lewis. 9 ed. Porto Alegre: Artmed, 2005.

LONARDONI, A. P.; GOULART, E.; OLIVEIRA, E. F. DE; ABELHA, M. C. F.. Feeding habitats and trophic overlap of the freshwater stingrays Potamotrygon falkneri and Potamotrygon motoro (Chondrichthyes, Potamotrygonidae) in the upper Paraná river floodplain, Brazil. Acta Scientiarum. Biological Sciences, Maringá, v.28, n.3, p.195202, 2006. DOI: https://doi.org/10.4025/actascibiolsci.v28i3.208

\section{LOLIS, S. F.. Macrófitas aquáticas do reservatório Luís}

Eduardo Magalhães (Lajeado)-TO: biomassa, composição da comunidade e riqueza de espécies. Tese (Doutorado em Ecologia de Ambientes Aquáticos Continentais) Universidade Estadual de Maringá, Maringá, 2008.

LUCINDA, P. H. F.; FREITAS, I. S.; SOARES, A. B.; MARQUES, E. E.; AGOSTINHO, C. S.; OLIVEIRA, R. J.. Fish, Lajeado Reservoir, rio Tocantins drainage, State of Tocantins, Brazil. Check List, Rio Claro, v.3, n. 2, p.77-90, 2007.

MARCUZZO, F. F. N.; GOULARTE, E. R. P.. Characterization of the Hydrological Year and Spatial Rains Mapping of Wet and Dry Periods in the State of Tocantins. Revista Brasileira de Geografia Física, Rio de Janeiro, v.6, n.1, p.091-099, 2013. DOI: https://doi.org/10.26848/rbgf.v06.1.p091-099

MARTIN, J. P.; BONAVENTURA, J.; FYHN, H.; FYHN, U. R. H.; GARLICK, R. L.; POWERS, D. A.. Estudo da estrutura e função das hemoglobinas isoladas das arraias da Amazônia do gênero Potamotrygon. Acta Amazônica, Manaus, v.8, n.4, p.173-184, 1978.

MARQUES, A. K.. Análise da diversidade fitoplanctônica no reservatório da Usina Hidroelétrica Luís Eduardo Magalhães, no médio Tocantins- TO: estrutura da comunidade, flutuações temporais e espaciais. Dissertação (Mestrado em Ciências do Ambiente) - Universidade Federal do Tocantins, Palmas, 2006. 
MARIANO, W. S.; OBA, E. T.; SANTOS, L. R. B.; FERNADES, M. N.. Respostas fisiológicas de jeju Hoplerythrinus unitaeniatus (Characiformes, Erythrinidae) expostos ao ar atmosférico. Revista Brasileira de Saúde e Produção Animal, Salvador, v.10, n.1, p.210-223, 2009.

MUUSZE, B.; MARCON J, V.T. G.; ALMEIDA-VAL V. M. F.. Hypoxia tolerance of Amazon fish: Respirometry and energy metabolism of the cichlid Astronotus ocellatus. Comp Biochem Physiol, Amsterdam, v.120, n.1, p.151-156, 1998. DOI: https://doi.org/10.1016/S1095-6433(98)10023-5

MCDONALD, G.; MILLIGAN, C. L.. Ionic, osmotic and acidbase regulation in stress. In: IWAMA, G. W.; PICKERING, A. D.; SUMPTER, J. P.; SCHRECK, C. B.. Fish stress and health in aquaculture. Cambridge: University Press, 1997. p.119-144.

OLIVEIRA, A. T.. Caracterização hematológica de Potamotrygon cf. histrix: subsídios ao manejo e conservação da espécie. Dissertação (Mestrado em Diversidade Biológica) - Universidade Federal do Amazonas, Manaus, 2008.

OLIVEIRA, A. T.. Parâmetros hematológicos, aspectos citoquímicos e ultraestruturais das células sanguíneas de três espécies de arraias (Chondricthyes: Potamotrygonidae) do Médio Rio Negro, Amazonas, Brasil. Tese (Doutorado em Diversidade Biológica) - Universidade Federal do Amazonas, Manaus, 2013.

OLIVER, S. L.; RIBEIRO, H.. Variabilidade climática e qualidade da água do Reservatório Guarapiranga. Sociedade e Ambiente, São Paulo, v.28, n.82, p.95-128, 2014. DOI: http://dx.doi.org/10.1590/S0103-40142014000300007.

PAVLIDIS, M.; FUTTER, W. C.; KATHARIOS, P.; DIVANACH, P.. Blood cell profile of six Mediterranean mariculture fish species. J. Appl. Ichthyol, Berlim, v.23, p.70-73, 2007. DOI: https://doi.org/10.1111/j.1439-0426.2006.00771.x

PAGLIARINI, C. D.; RIBEIRO, C. S.; SPADA, L.; DELARIVA, R. L.; CHAGAS, J. M. A.; ANJOS, L. A.; RAMOS, I. P.. Trophic ecology and metabolism of two species of nonnative freshwater stingray (Chondrichthyes: Potamotrygonidae). Hydrobiologia, Netherlands, v.847, p.2895-2908, 2020. DOI: https://doi.org/10.1007/s10750-020-04283-1

PINTO, P. H. P.. As chuvas no Estado do Tocantins: distribuição geográfica e gênese das variações rítmicas. Dissertação (Mestrado em Geografia) - Universidade Estadual Paulista Júlio de Mesquita Filho, Rio Claro, 2013.

PINTO, M. S.; DÓRIA, C. R. C.; MARQUES, E. E.. Alterações temporais sobre a estrutura funcional das assembleias de peixes durante onze anos de formação de um reservatório do médio rio Tocantins, Brasil. Biota Amazônia, Macapá, v.9, n.1, p.17-21, 2019. DOI: http://dx.doi.org/10.18561/21795746/biotaamazonia.v9n1p17-21

POTTINGER, T. G.; CALDER, G. M.. Physiological stress in fish during toxicological procedures: A potentially confounding factor. Environ. Toxicol. Water Qual., Ottawa, v.10, n.2, p.135-146, 1995. DOI: https://doi.org/10.1002/tox.2530100208
RANDALL, D.; BURGGREN, W.; FRENCH, K.. Fisiologia animal, mecanismos e adaptações. 4 ed. São Paulo: Guanabara Koogan. S. A, 2006.

RIBEIRO, M. C. L. B.; PETRERE, M.; JURAS, A. A.. Ecological Integrity and Fisheries Ecology of the Araguaia-Tocantins River Basin, Brazil. Regul. Rios: Res. Mgmt., São Luís, v.11, n.3-4, p.325-350, 1995. DOI:

https://doi.org/10.1002/rrr.3450110308

RINCON, G.. Aspectos taxonômicos, alimentação e reprodução da raia de água doce Potamotrygon orbignyi (Castelnau) (Elasmobranchii: Potamotrygonidae) no rio Paranã-Tocantins. Tese (Doutorado em Ciências Biológicas Zoologia) - Universidade Estadual Paulista Júlio de Mesquista Filho, Rio Claro, 2006.

ROLDÃO, A. F.; FERREIRA, V. O.. Climatologia do Estado do Tocantins/Brasil. Caderno de Geografia, Belo Horizonte, v.29, n.59, p.1161-1181, 2019. DOI: https://doi.org/10.5752/P.2318-2962.2019v29n59p1161

ROSA, R. S.; CHARVET-ALMEIDA, P.; QUIJADA, C. C. D.. Biology of the South American Potamotrygonid Stingrays. In: CARRIER, J. F.; MUSICK, J. A.; HEITHAUS, M. R.. Sharks and their relatives II: biodiversity, adaptive physiology, and conservation. Washington: CRC Press, 2010. p.241-286.

ROSA, R. S.. A systematic revision of the South American freshwater stingrays (Chondrichthyes: Potamotrygonidae). Thesis (Doctorate) - College of William and Mary, Williamsburg, 1985.

SANTOS, J. M.; SANTOS J. C.; MARQUES, E. E.; ARAÚJO, G. C.; SEIBERT, C. S. LOPES-FERREIRA, M.; LIMA, C.. Stingray (Potamotrygon rex) maturity is associated with inflammatory capacity of the venom. Toxicon, London, v.163, p.74-83, 2019. DOI: https://doi.org/10.1016/j.toxicon.2019.03.013

SHAPIRO, S. S.; WILK, M. B.. An analysis of variance test for normality (complete samples). Biometrika, v.52, n.3/4, p.591-611, 1965. DOI: https://doi.org/10.2307/2333709

SHIBUYA, A.; ZUANON, J.; ARAÚJO, M. L. G.; TANAKA, S.. Morphology of lateral line canals in Neotropical freshwater stingrays (Chondrichthyes: Potamotrygonidae) from Negro River, Brazilian Amazon. Neotropical Ichthyology, Porto Alegre, v.8, n.4, p.867-876, 2010. DOI: https://doi.org/10.1590/S1679-62252010000400017

SIMPLENDORFER, C. A.; HEUPEL M. R.; WHITE W. T.; DULVY N. K.. The importance of research and public opinion to conservation management of sharks and rays: a synthesis. Marine and Freshwater Research, Camberra, v.62, n.6, p.518-527, 2011. DOI: https://doi.org/10.1071/MF11086

SPEERS-ROESCH, B.; RICHARDS, J. G.; BRAUNER, C. J.; FARRELL, A. P.; HICKEY, A. J. R.; WANG, Y. S.; RENSHAW, G. M.V.. Hypoxia tolerance in elasmobranchs. I. Critical oxygen tension as a measure of blood oxygen transport during hypoxia exposure. The Journal of Experimental Biology, London, v.215, p.93-102, 2012. DOI: https://doi.org/10.1242/jeb.059642

SPEERS-ROESCH, B.; TREBERG, J. R.. The unusual energy metabolism of elasmobranch fishes. Comp. Biochem. 
Physiol. Mol. Integr. Physiol., Washington, v.155, n.4, p.417434, 2010. DOI: https://doi.org/10.1016/i.cbpa.2009.09.031

SOUZA, L. B.. Ritmo climático e tipos de tempo no Estado do Tocantins, Brasil. In: SIMPÓSIO BRASILEIRO DE CLIMATOLOGIA GEOGRÁFICA, 12. Anais. Goiânia: Associação Brasileira de Climatologia, 2016.

TAVARES-DIAS, M.; MORAES, F. R.. Hematological parameters for the Brycon orbignyanus, 1850 (Osteichthyes: Characidae) intensively bred. Hidrobiológica, Cidade el México, v. 16, n.3, p.273-276, 2006.

TAVARES-DIAS, M.; OLIVEIRA, M. S. B.; GONÇALVES, R. A.; SILVA, L. M. A.. Ecology and seasonal variation of parasites in wild Aequidens tetramerus, a Cichlidae from the Amazon. Acta Parasitologica, Germany, v.59, n.1, p.158-164, 2014. DOI: https://doi.org/10.2478/s11686-014-0225-3

TUNDISI, J. G.; MATSUMURA-TUNDISI, T.. Limnologia. São Paulo: Oficina de Textos, 2008.

VAL, A. L.; AFFONSO, E. G.; ALMEIDA-VAL, V. M. F.. Adaptive features of Amazon fishes: blood characterísticas of curimatã (Prochilodus cf. Nigricans, Osteichthyes).

Physiological Zoology, Chicago, v.65, n.4, p.832-843, 1992. DOI: https://doi.org/10.2307/30158542

VAL, A. L.; SILVA, M. N. P.; ALMEIDA-VAL, V. M. F.. Hypoxia adaptation in fish of the Amazon: a never-ending task, South African Journal of Zoology, Luanda, v.33, n.2, p.107-114, 1998. DOI:

https://doi.org/10.1080/02541858.1998.11448459

VASCONCELOS, H. C. G.; OLIVEIRA, J. C. S. Alimentação de Potamotrygon motoro (Chondrichthyes, Potamotrygonidae) na planície de inundação da APA do Rio Curiaú, MacapáAmapá-Brasil. Biota Amazônia, Macapá, v.1, n.2, p.66-73, 2011. DOI: http://dx.doi.org/10.18561/21795746/biotaamazonia.v1n2p66-73

ZENI, T. O.; OSTRENSKY, A.; WESTPHAL, G. G. C.. Respostas adaptativas de peixes a alterações ambientais de temperatura e de oxigênio dissolvido. Archives of Veterinary Science, Curitiba, v.21, n.3, p.01-16, 2016. DOI: http://doi.org/10.5380/avs.v21i3.40165

A CBPC - Companhia Brasileira de Produção Científica (CNPJ: 11.221.422/0001-03) detém os direitos materiais desta publicação. Os direitos referem-se à publicação do trabalho em qualquer parte do mundo, incluindo os direitos às renovações, expansões e disseminações da contribuição, bem como outros direitos subsidiários. Todos os trabalhos publicados eletronicamente poderão posteriormente ser publicados em coletâneas impressas sob coordenação da Sustenere Publishing, da Companhia Brasileira de Produção Científica e seus parceiros autorizados. Os (as) autores (as) preservam os direitos autorais, mas não têm permissão para a publicação da contribuição em outro meio, impresso ou digital, em português ou em tradução. 